\title{
A Meta-Model Based Approach for Rapid Formability Estimation of Continuous Fibre Reinforced Components
}

\author{
Clemens Zimmerling ${ }^{1, a)}$, Dominik Dörr ${ }^{1}$, Frank Henning ${ }^{1,2}$, Luise Kärger ${ }^{1}$ \\ ${ }^{1}$ Karlsruhe Institute of Technology (KIT), Institute of Vehicle System Technology, Karlsruhe, Germany \\ ${ }^{2}$ Fraunhofer - Institute of Chemical Technology (ICT), Pfinztal, Germany \\ ${ }^{a)}$ Corresponding author: clemens.zimmerling@kit.edu
}

\begin{abstract}
Due to their high mechanical performance, continuous fibre reinforced plastics (CoFRP) become increasingly important for load bearing structures. In many cases, manufacturing CoFRPs comprises a forming process of textiles. To predict and optimise the forming behaviour of a component, numerical simulations are applied. However, for maximum part quality, both the geometry and the process parameters must match in mutual regard, which in turn requires numerous numerically expensive optimisation iterations. In both textile and metal forming, a lot of research has focused on determining optimum process parameters, whilst regarding the geometry as invariable. In this work, a meta-model based approach on component level is proposed, that provides a rapid estimation of the formability for variable geometries based on pre-sampled, physics-based draping data. Initially, a geometry recognition algorithm scans the geometry and extracts a set of doubly-curved regions with relevant geometry parameters. If the relevant parameter space is not part of an underlying data base, additional samples via Finite-Element draping simulations are drawn according to a suitable design-table for computer experiments. Time saving parallel runs of the physical simulations accelerate the data acquisition. Ultimately, a Gaussian Regression meta-model is built from the data base. The method is demonstrated on a box-shaped generic structure. The predicted results are in good agreement with physics-based draping simulations. Since evaluations of the established meta-model are numerically inexpensive, any further design exploration (e.g. robustness analysis or design optimisation) can be performed in short time. It is expected that the proposed method also offers great potential for future applications along virtual process chains: For each process step along the chain, a meta-model can be set-up to predict the impact of design variations on manufacturability and part performance. Thus, the method is considered to facilitate a lean and economic part and process design under consideration of manufacturing effects.
\end{abstract}

\section{INTRODUCTION}

Continuous fibre reinforced plastics (CoFRP) exhibit remarkable mechanical properties whilst offering significant weight saving potentials due to their low density. However, designing CoFRP components is often challenging: Apart from the geometry, further variables need to be defined for optimum part performance, e.g. fibre orientation or the stacking sequence. Additionally, process parameters, such as blank holder forces during draping, need to be adjusted for maximum part quality. Efforts have been made for structural optimisation and for process optimisation, e.g. in [1] or [5], respectively. However, most of the works focus on either structural or process optimisation, although [8] indicates, that process optimisation can have significant impact on the structural performance. Additionally, most process optimisation strategies in both textile and metal forming disregard geometry variations and assume a fixed design [1-3]. While process optimisation certainly improves the part quality to a certain extent, it cannot entirely compensate for an arbitrarily adverse design. Hence, accounting for manufacturability already during the design phase of a component offers great potential for cost saving and prevents expensive re-design loops. In general, a combined process-structure-optimisation including variable geometries is desirable and may be perceived as the overall goal of ongoing research in CoFRP design.

Manufacturing of CoFRP components typically requires multiple steps, often including a forming process of textiles (draping). To predict the draping behaviour, many researchers have focused on appropriately describing the forming process in Finite-Element (FE) simulations, e.g. [6,7]. Moreover, simulating and connecting each CoFRPmanufacturing step to a virtual process chain is increasingly aspired [4,8]. However, due to their highly non-linear 
nature, FE-analyses quickly become time-consuming and for iterative optimisations the computation time becomes impracticably long. Consequently, time-efficiency is a key factor in process and part design strategies for CoFRP.

One option to time-efficiently account for manufacturing during part design is the use of simplified, possibly even analytical relations, such as kinematic draping approaches for textile forming [9] or geodesic designs for filament winding. Typically these relations neglect the actual physical behaviour (e.g. material non-linearities, frictional effects or process conditions) and are thus of limited accuracy. Additionally, most of these models do not return information to the designer on how to modify geometric features (e.g. fillet radii, draft angles) to improve the design.

As an option for time-efficient process optimisation, machine learning techniques have been successfully applied in the field of metal forming [2-3]: Instead of explicitly formulating and solving a constitutive relationship for the forming process, an easy-to-evaluate phenomenological relationship (meta-model) between input (process parameter) and observed output (part quality measure) is established ('learned'). Optimisation is subsequently performed on the meta-model in short time.

In this work, machine learning techniques are applied to rapidly assess geometric features of a component with respect to formability: A meta-model is trained to describe the relation between variable geometries (input) and corresponding shear angles during forming (output). As an initial evaluation of the approach, a symmetric box-shaped geometry is studied and its draping behaviour is predicted using meta-models. This rapid formability estimation may be perceived as a first step towards machine learning assisted part and process design.

\section{MODELLING APPROACH}

\section{General Approach}

From the perspective of meta-modelling, a numerical simulation of a forming process with focus on geometric features can be interpreted as a function $\varphi: G \rightarrow \mathrm{P}$, that maps from a set of geometric features $\underline{g} \in G$ to a resulting product attribute $\underline{\rho} \in \mathrm{P}$ (shear angles). Since the evaluation of $\varphi$ is costly (FE-simulation), an easy-to-evaluate function for the meta-model $\mu: G \rightarrow \mathrm{P}$ is trained as an approximation for $\varphi$ from $n$ observations in a data set $D^{n}$, i.e. $D^{n}=$ $\left\{(\underline{g}, \underline{\rho})_{i=1 \ldots n}\right\}$. The meta-model function $\mu$ is preselected from the function class $\mathcal{M}$. Commonly used function classes for machine learning are polynomials, splines, neural networks, support vector machines, decision trees or Gaussian Regression (GR) [10]. Being a universal approximator in conjunction with probability theory, GR aims at identifying the statistically most likely relationship between input and corresponding output data. It has shown remarkable predictive capabilities in engineering applications, see e.g. [3], and is also used in this work.

GR applies Bayesian statistics theory and uses multivariate Gaussian distributions as a prior probability. The prior is usually defined with a zero or constant mean and the covariance matrix $\underline{\underline{K}}$. The elements of $\underline{\underline{K}}$ are the covariance functions $K_{i j}$ and are often chosen as squared exponential functions. A one-dimensional example for a random variable $x$ and an observed data point $\hat{x}$ is given by

$$
K_{x \widehat{x}}=\sigma_{0}^{2} \exp \left(-\frac{1}{2}\left(\frac{x-\hat{x}}{\lambda}\right)^{2}\right),
$$

in which the hyperparameters $\sigma_{0}$ and $\lambda$ are the maximum allowable covariance of the observed data and the length scale of the random variable, respectively. During training these hyperparameters are optimised to fit the data. As is readily seen, equation ( 1 ) follows the notion that close points in the input space (i.e. $|x-\hat{x}| \rightarrow 0$ ) lead to similar results and remote points $(|x-\hat{x}| \rightarrow \infty)$ do not influence each other. Details on GR can be found in [11]. Subsequently, the logarithmic likelihood $\ln (p(\underline{\rho} \mid \underline{g}))$ is maximised during the training of the model:

$$
\ln (p(\underline{\rho} \mid \underline{g}))=-\frac{1}{2} \underline{\rho}^{T}\left(\underline{\underline{K}}^{-1}\right) \underline{\rho}-\frac{1}{2} \ln (\operatorname{det}(\underline{\underline{K}}))-\frac{n}{2} \ln (2 \pi) \stackrel{!}{\rightarrow} \max
$$

The workflow proposed in this work is schematically shown in Fig. 1. The overall aim is to predict the draping result for a geometry with an arbitrary number of doubly-curved regions $k$ by a meta-model $\mu$, which has solely been trained with generic draping-examples of sub-structures. Initially, the geometry of interest is scanned and a set of parameters $\underline{g}$ for each of the $k$ relevant doubly-curved regions is extracted and stored in a geometry set $G^{k}=\left\{\underline{g_{1}}, \ldots, \underline{g_{k}}\right\}$. If the identified geometry parameters lie outside the range of the previously sampled data set $D^{n}$ (i.e. $\exists \underline{g}: \underline{g} \notin D^{n}$ ) or if no sampling has been performed so far (initial run), then data sampling is performed: According to a Design of Experiments (DoE) the data set is generated or extended by means of draping simulations. Thus, the data set $D^{n}$ can be thought of as a catalogue of geometry-specific draping examples. In this study, space- 
filling DoEs have been combined with classical DoEs: Latin Hypercube Sampling (LHS) is used to explore the parameter space, whilst a full-factorial and a Box-Behnken-Design sample the boundary of the parameter space. For each of the $n$ simulations a characteristic results vector $\rho_{1} \ldots n$ is determined. The geometric parameters and the results vector form pairs of values $(g, \rho)_{1 \ldots n}$ and are stored in the data set $D^{n}=\{(\boldsymbol{g}, \boldsymbol{\rho})\}$. Training of the GR-model is performed using the sampled data set $D^{n}$ by maximizing equation ( 2 ) and once the training is completed, predictions on the effect of local geometry variations can be made.

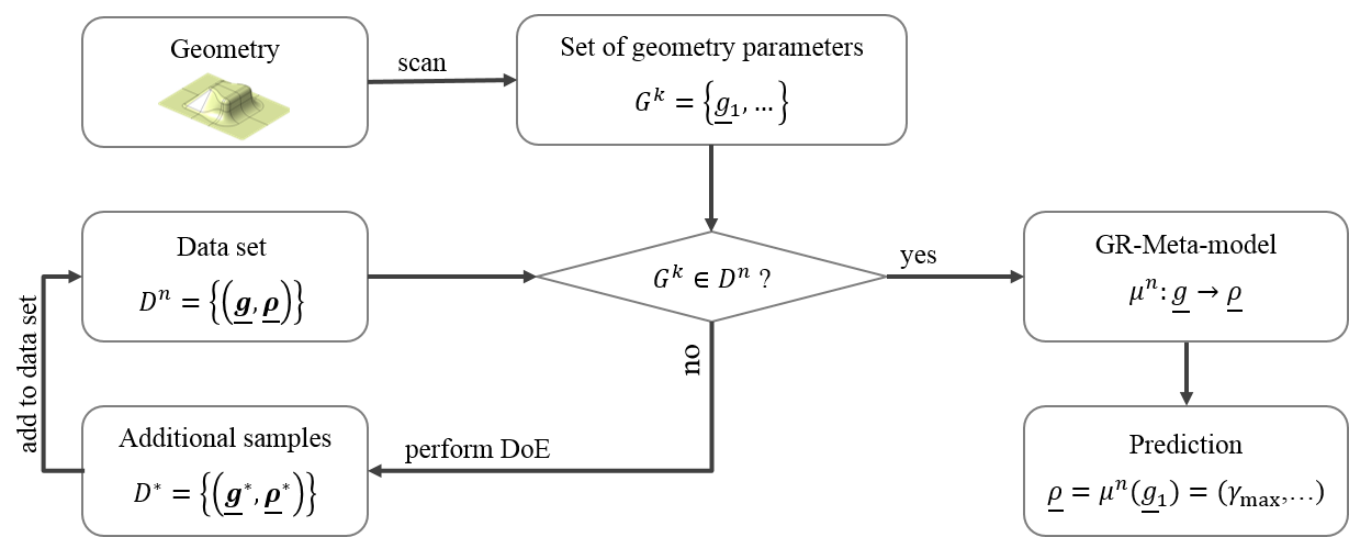

FIGURE 1. Schematic workflow for a meta-model based formability assessment of CoFRP

For CoFRP a frequently used criterion to assess the draping quality is the in-plane shear angle $\gamma_{12}$. Since doublecurved regions evoke concentrated shear deformations, they limit the manufacturability of the part and are thus of particular interest. Therefore, the approach concentrates on identifying doubly-curved regions, (see examples in Fig. 2 on the right) and predicting the corresponding shear angle distribution. For initial demonstration, the mutual influence of neighbouring regions is eliminated by using a box shaped test-geometry, where the corners are sufficiently distant $(\geq 50 \mathrm{~mm}$ ). Two example geometries are presented in Fig. 5 for visualisation.

\section{Acquisition of the Data}

\section{Geometric parameterisation for a box shaped geometry}

In this work, the above outlined workflow is applied to predict the shear angle distribution during draping of a box-shaped geometry, whose corners provoke strong shear deformations. In order to build a data set $D^{n}$, a parametric geometry for the corners is defined, whose set of geometric features $g$ can be adjusted to generate various shapes. The parameterisation scheme can be found in Fig. 2 (left) along with the used parameter range (centre). Possible resulting geometries are a semi-sphere, pyramids or cuboids and their variations. Some examples for visualisation are given in the right of Fig. 2. For demonstration purposes, this work focuses on one parameterisation scheme.

\begin{tabular}{c|c|c|c|} 
Parameterisation scheme & Symbol & Meaning & Range \\
\hline & $r_{1}$ & Radius (top) & $5 \ldots 40 \mathrm{~mm}$ \\
& $r_{2}$ & Radius (bottom) & $5 \ldots 40 \mathrm{~mm}$ \\
$\alpha$ & Draft angle & $5 \ldots 40^{\circ}$ \\
& $\Theta_{\mathrm{AR}}=\frac{w}{l}$ & Aspect ratio & $1.0 \ldots 2.0$ \\
$w=A R l$ & Width & $50 \ldots 600 \mathrm{~mm}$ \\
$h$ & Height & $50 \mathrm{~mm}$
\end{tabular}

FIGURE 2. Parameterisation scheme, the evaluated parameter range and example geometries for visualisation. 


\section{Draping simulation and data processing}

According to the DoE described above, sample geometries and according tool geometries are generated. The forming simulations are performed using the commercial FE-solver ABAQUS within a forming simulation framework in-house developed at KIT-FAST [6-8]. This framework includes a fully automatised pre- and postprocessing as well as several constitutive models for intra-ply and inter-ply deformation mechanisms implemented in user-subroutines $[7,8]$. The used material parameters in this work are of generic nature. A constant in-plane shear modulus of $G_{12}=$ $1 \mathrm{MPa}$ in combination with a hyperelastic constitutive equation is used, showing a shear locking angle of $58^{\circ}$. To study the isolated draping behaviour and to eliminate additional effects, such as inter-ply slippage, only one ply is draped. For reduced computational effort, the symmetry of the model is employed by simulating only a quarter-model. The fibre orientations are $0^{\circ} / 90^{\circ}$ and $\pm 45^{\circ}$, respectively, with respect to the $x$-axis in Fig. 3 a).

An exemplary simulation setup and draping result is given in Fig. 3 a) and b). The resulting shear angles are collected in a vector $\underline{\gamma}_{12}=\left(\gamma_{12}^{1}, \ldots, \gamma_{12}^{i}, \ldots, \gamma_{12}^{\mathrm{n}}\right)^{T}$, where $\gamma_{12}^{i}$ denotes the in-plane shear angle of the $i$-th element. To describe the shear angle distribution, the elements of $\underline{\gamma}_{12}$ are sorted into bins of $10^{\circ}$ in a histogram (cf. Fig. $3 \mathrm{c}$ )). From this histogram statistical central moments are computed to efficiently describe the distribution. In this work, the maximum absolute shear angle $\left|\gamma_{12}\right|_{\max }$, the average shear angle $\overline{\gamma_{12}}$, the standard deviation $\sigma_{\gamma 12}=\sqrt{\operatorname{var}\left(\underline{\gamma}_{12}\right)}$, and the higher-order statistical moments skewness $v\left(\underline{\gamma}_{12}\right)=\operatorname{skew}\left(\underline{\gamma}_{12}\right)$ and kurtosis $w\left(\underline{\gamma}_{12}\right)=\operatorname{kurt}\left(\underline{\gamma}_{12}\right)$ are stored in the results vector $\rho$. The skewness can be interpreted as how much the histogram 'leans' to the left $(v>0)$ or right $(v<$ 0 ) from the mean, while the kurtosis describes the sharpness $(w \rightarrow-\infty)$ or flatness $(w \rightarrow \infty)$ of the peak of the histogram. Clearly, optimising the draping result aims at bringing all shear angles as close as possible to zero. In terms of statistical moments, this means $\left|\gamma_{12}\right|_{\max } \rightarrow \min , \overline{\gamma_{12}} \rightarrow \min , \sigma_{\gamma 12} \rightarrow \min , v\left(\underline{\gamma_{12}}\right) \rightarrow \max , w\left(\underline{\gamma_{12}}\right) \rightarrow \min$.

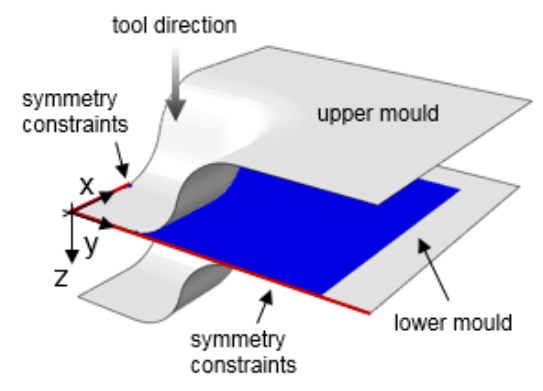

a)

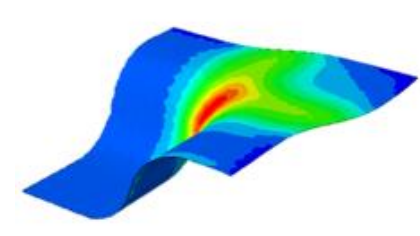

b)

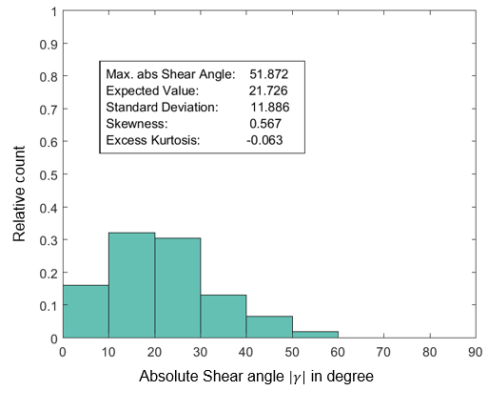

c)

FIGURE 3. Visualisation of the a) draping simulation setup, b) qualitative plot of the resulting shear angles $\gamma_{12}$ for $0^{\circ}$ fibre orientation, c) histogram of the relative shear angle distribution

\section{Training and Validating the Meta-Model Quality}

Prior to training the meta-model, $n$ draping simulations with known geometric parameters $g_{i=1 \ldots n}=$ $\left(r_{1}, r_{2}, \alpha, w, l\right)_{i=1 \ldots n}$ are performed, each yielding a result vector $\underline{\rho}_{1 \ldots n}=\left(\left|\gamma_{12}\right|_{\max }, \overline{\gamma_{12}}, \sigma_{\gamma 12}, v\left(\underline{\gamma_{12}}\right), w\left(\underline{\gamma_{12}}\right)\right)_{i=1 \ldots . .}$. Both are combined to form pairs of values $(\underline{g}, \underline{\rho})_{i=1 \ldots n}$ that are stored in the data set $D^{n}=\{(\underline{\boldsymbol{g}}, \underline{\boldsymbol{\rho}})\}$, according to the flowchart diagram in Fig. 1. From this database a GR-meta-model is trained. Additionally, a linear (LP) and quadratic polynomials (QP) are trained for performance comparison. In general, the more data samples are used for training, the more accurate the model's predictions on new data will be. In this work, $n=71$ simulations are used to train the model. To measure the resulting prediction quality of the meta-model, leave-one-out cross validation (CV) is performed. Beyond that, a test set with unseen data is generated. The results are given in Fig. 4 a) and indicate a high accuracy of the GR: For example the root mean squared error (RMSE) for predicting $\left|\gamma_{12}\right|_{\max }$ is less than one degree. Also a predicted-vs-actual plot is given in Fig. 4 b) showing a superior performance of the GR: The closer the markers lie to the dashed line, the more reliable the meta-model is. While significant deviations are observed for LP and QP, the GR is able to accurately predict the shear angle. 


\begin{tabular}{c|c|ccc}
\multicolumn{2}{c|}{} & \multicolumn{3}{c}{ RMSE } \\
\cline { 3 - 5 } \multicolumn{2}{c}{} & LP & QP & GR \\
\hline \multirow{2}{*}{$\left|\gamma_{12}\right|_{\text {max }}$} & CV & 5.59 & 5.92 & 1.85 \\
& Test & 3.49 & 3.12 & 0.85 \\
\hline \multirow{2}{*}{$\gamma_{12}$} & CV & 1.94 & 1.81 & 1.89 \\
& Test & 3.21 & 3.06 & 2.13 \\
\hline \multirow{2}{*}{$\sigma_{\gamma 12}$} & CV & 1.65 & 1.70 & 1.70 \\
& Test & 1.06 & 1.02 & 0.49 \\
\hline \multirow{2}{*}{$v\left(\underline{\gamma}_{12}\right)$} & CV & 0.23 & 0.23 & 0.21 \\
& Test & 0.14 & 0.17 & 0.21 \\
\hline \multirow{2}{*}{$w\left(\underline{\gamma}_{12}\right)$} & CV & 0.41 & 0.42 & 0.41 \\
& Test & 0.32 & 0.32 & 0.22
\end{tabular}

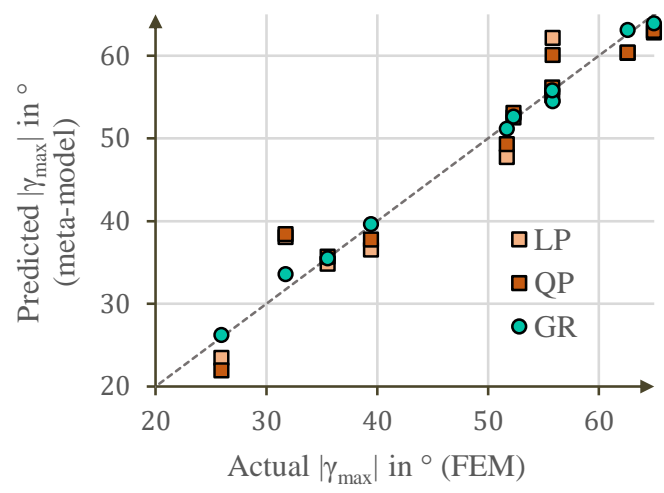

FIGURE 4. Performance evaluation of Gaussian Regression (GR) meta-models compared to linear (LP) and quadratic polynomials (QP).

\section{APPLICATION TO TEST GEOMETRY}

The proposed approach is tested by predicting the shear deformations during draping of two complex curved box geometries, shown at the top in Fig. 5, and for two fibre orientations (FO). The geometries are symmetric and show two doubly-curved regions, denoted by corner 1 and corner 2 . These corners fall into the aforementioned parameterisation scheme. It has to be noted, that the parameters have not been used for sampling and thus, the previously trained meta-model must make predictions on new, unseen data. In a first step, a self-written algorithm identifies the doubly curved regions and determines the corresponding geometry parameters. Subsequently, the metamodel predicts the shear angles based on the identified geometry parameters. To validate the predictions of the metamodel, FE-simulations for the two test geometries are performed. Both results are provided in the column diagram for $\left|\gamma_{12}\right|_{\text {max }}$ in Fig. 5 and show a good general agreement. The maximum deviation for $0^{\circ} \mathrm{FO}$ is $2.09^{\circ}$, while for $45^{\circ} \mathrm{FO}$ one outlier is found $\left(6.55^{\circ}\right.$ deviation for corner 2 of geometry 2$)$. The remaining predictions match the FE-results very well with only $1.92^{\circ}$ maximum deviation. Similarly good agreements are found for $\overline{\gamma_{12}}, \sigma_{\gamma 12}, v\left(\underline{\gamma_{12}}\right)$ and $w\left(\underline{\gamma_{12}}\right)$.

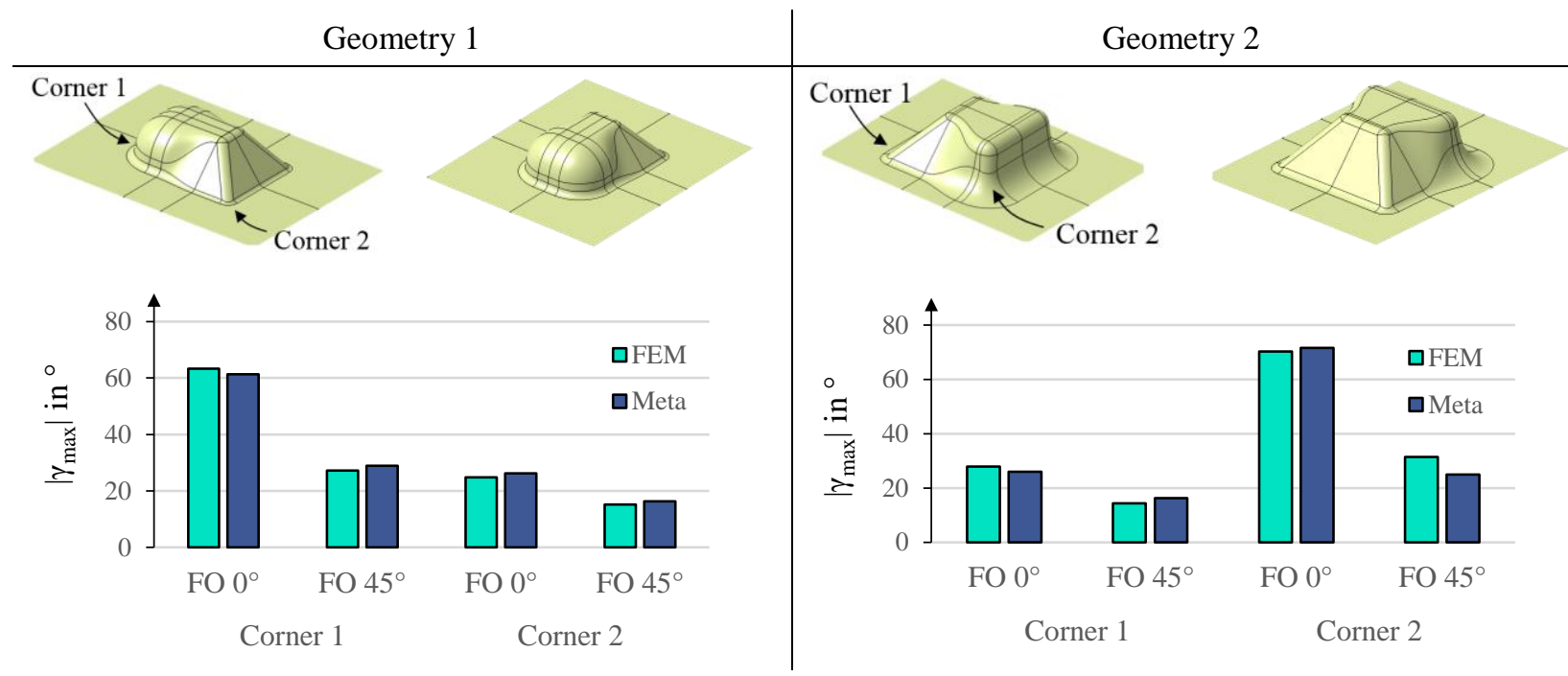

FIGURE 5. Application of the GR meta-model to box-shaped test geometries and comparison of the predicted maximum absolute shear angle $\left|\gamma_{12}\right|_{\max }$ to corresponding FE-results. 


\section{DISCUSSION AND CONCLUSION}

The proposed approach suggests the prediction of draping results for variable geometries by evaluating pre-trained meta-models rather than solving numerically expensive FE-simulations. As shown in this work, the physics-based draping behaviour can be learned and predicted for variable geometries with good accuracy. Thereby a rapid estimation of the forming result is made directly from geometric information. This enables a quick effect assessment of design variations onto the formability. 71 simulation examples are found to suffice for a successful prediction of the shear angle distribution in doubly-curved regions of a complexly curved, box-shaped geometry. Non-parametric GR-models outperform classical polynomial approaches in terms of prediction accuracy. Withal the proposed approach is not restricted to draping prediction but can be applied to other process simulations (e.g. curing). Thus, it offers great potential for application along virtual process chains $[4,8]$.

Important subjects for future research are the modelling of the mutual influence between two neighbouring regions, which has been eliminated in this demonstration. Furthermore, the inclusion of additional parameterisation schemes to generalise from box-shaped geometries to arbitrary part designs is crucial for further development. One option may be the use of convolutional neural networks (CNN): [12] shows that CNNs are able to learn physical effects from generic example data and generalise to real-world engineering problems, provided the underlying training data set is sufficiently large. Ultimately, the proposed approach could enable a machine-learning assisted tool for engineers to provide fast and physics-based performance evaluations at early stages during the design phase.

\section{ACKNOWLEDGMENTS}

The authors would like to thank the German Federal State Ministry of Science, Research and the Arts of Baden-Württemberg (MWK) for funding the project "Forschungsbrücke Karlsruhe-Stuttgart", which the presented work is carried out for. Additionally, the authors would like to thank the project partners at the Institute of Aircraft Design (IFB) of Stuttgart University for collaboration and discussion. The work is also part of the Young Investigator Group (YIG) "Tailored Composite Materials for Lightweight Vehicles", generously funded by the Vector Stiftung.

\section{REFERENCES}

1. S. Chen, L. T. Harper, A. Endruweit, N. A. Warrior, "Formability optimisation of fabric preforms by controlling material draw-in through in-plane constraints“, in Composites Part A (76, 2015), pp. 10-19.

2. M.H.A. Bonte, A. H. van den Boogaard, J. Huétink, "A Metamodel Based Optimisation Algorithm for Metal Forming Processes" in Advanced Methods in Material Forming (Springer, Berlin/Heidelberg, 2007), pp. 55-72.

3. J. Jakumeit, M. Herdy, M. Nitsche, ,Parameter optimization of the sheet metal forming process using an iterative parallel Kriging algorithm“ in Structural and Multidisciplinary Optimization, (29, 2005), pp. 498-507.

4. L. Kärger, A. Bernath, F. Fritz, S. Galkin, D. Magagnato, A. Oeckerath, A. Schön, F. Henning, "Development and validation of a CAE chain for unidirectional fibre reinforced composite components" in Composite Structures $(132,2015)$, pp. 350-358.

5. G. Allaire and G. Delgado, "Stacking sequence and shape optimization of laminated composite plates via a levelset method" in Journal of the Mechanics and Physics of Solids, (97, 2016), pp. 168-196.

6. D. Dörr, F. Schirmaier, F. Henning, L. Kärger, "A viscoelastic approach for modeling bending behavior in Finite Element forming simulation of continuously fibre reinforced composites" in Composites Part A, (94, 2017), pp. 113-123.

7. F.J. Schirmaier, D. Dörr, F. Henning, L. Kärger, A macroscopic approach to simulate the forming behaviour of stitched unidirectional non-crimp fabrics (UD-NCF) in Composites Part A, 102, (2017), pp. 322-335.

8. L. Kärger, S. Galkin, C. Zimmerling, D. Dörr, J. Linden, A. Oeckerath, K. Wolf,: "Forming optimisation embedded in a CAE chain to assess and enhance the structural performance of composite components", Composite Structures, in submission.

9. C. Mack, H.M. Taylor, "The fitting of woven cloth to surfaces", J. of the Textile Institute (47, 1956), pp. 477-488.

10. T. W. Simpson, J. Poplinski, P. N. Koch, J. K. Allen, "Metamodels for computer-based engineering design: survey and recommendations" Engineering with computers, (17, 2001), pp. 129-150.

11. C.E. Rasmussen, C.K. Williams, "Gaussian processes for machine learning”, Cambridge: MIT press, 2006

12. X. Guo, W. Li, F. Iorio, "Convolutional neural networks for steady flow approximation", in Proceedings of the 22nd International Conference on Knowledge Discovery and Data Mining, 2016, pp. 481-490. 